Published in final edited form as:

Med Image Anal. 2007 October ; 11(5): 492-502.

\title{
Delineating White Matter Structure in Diffusion Tensor MRI with Anisotropy Creases
}

\author{
Gordon Kindlmann ${ }^{\mathrm{a}}$, Xavier Tricoche ${ }^{\mathrm{b}}$, and Carl-Fredrik Westin ${ }^{\mathrm{a}}$ \\ aLaboratory of Mathematics in Imaging, Department of Radiology, Harvard Medical School, USA \\ bScientific Computing and Imaging Institute, University of Utah, USA
}

\begin{abstract}
Geometric models of white matter architecture play an increasing role in neuroscientific applications of diffusion tensor imaging, and the most popular method for building them is fiber tractography. For some analysis tasks, however, a compelling alternative may be found in the first and second derivatives of diffusion anisotropy. We extend to tensor fields the notion from classical computer vision of ridges and valleys, and define anisotropy creases as features of locally extremal values of tensor anisotropy. Mathematically, these are the loci where the gradient of anisotropy is orthogonal to one or more eigenvectors of its Hessian. We propose that anisotropy creases provide a basis for extracting a skeleton of the major white matter pathways, in that ridges of anisotropy coincide with interiors of fiber tracts, and valleys of anisotropy coincide with the interfaces between adjacent but distinctly oriented tracts. The crease extraction algorithm we present generates high-quality polygonal models of crease surfaces, which are further simplified by connected-component analysis. We demonstrate anisotropy creases on measured diffusion MRI data, and visualize them in combination with tractography to confirm their anatomic relevance.
\end{abstract}

\section{Keywords}

Diffusion Tensor MRI; anisotropy; ridges and valleys; crease surface extraction; white matter geometry

\section{Introduction}

Diffusion tensor magnetic resonance imaging (DTI) has become a popular means of measuring the structure of white matter in the central nervous system (Basser et al., 1994; Pierpaoli et al., 1996; Basser and Jones, 2002). The coherent organization of axons on nerve bundles contributes to the magnitude and orientation of diffusion anisotropy (Beaulieu, 2002). To the extent that a single diffusion tensor model captures the diffusive behavior in the underlying tissue, image processing of tensor-valued data can be leveraged to analyze the macroscopic architecture of the white matter in disease and in health. The de facto standard measure of microstructural tissue organization, calculated from the diffusion tensor, is fraction anisotropy, or FA (Basser, 1995). Many applications of DTI are based on region-of-interest measurements of FA, guided by either by prior knowledge of neuroanatomy, or by measurements from functional MRI (Klingberg et al., 2000; Kubicki et al., 2003; Kanaan et al., 2005; Salat et al., 2005; Tuch et al., 2005).

\footnotetext{
Publisher's Disclaimer: This is a PDF file of an unedited manuscript that has been accepted for publication. As a service to our customers we are providing this early version of the manuscript. The manuscript will undergo copyediting, typesetting, and review of the resulting proof before it is published in its final citable form. Please note that during the production process errors may be discovered which could affect the content, and all legal disclaimers that apply to the journal pertain.
} 
Fiber tractography is another common DTI analysis method, in which the course of axons in fiber tracts is approximated by path integrals along the direction of greatest diffusivity, the diffusion tensor principal eigenvector (Basser et al., 2000). Neuroscientific studies can then be based upon measurements of tract geometry (Dougherty et al., 2005) or of tensor attributes along tracts (Corouge et al., 2006). Further post-processing of tractography can involve clustering coherent groups of similar tracts into models of major fiber pathways (Zhang et al., 2006; O'Donnell et al., 2006). The combination of tractography and clustering algorithms requires a non-trivial number of parameter settings, which may affect their practical application to neuroscientific studies (Moberts et al., 2005). This has motivated our exploration of modeling techniques that work more directly with the underlying tensor data and its attributes.

In two-dimensional images and height fields, ridges and valleys, collectively referred to as creases, have been an object of study for many years in different disciplines. In the context of geomorphology, de Saint-Venant (1852) defines creases as the loci where the slope is minimal along the isocontours of the relief, which was later reformulated in terms of the Hessian of the height function by Haralick (1983). Maxwell (1870) gives a topological and global definition of ridges and valleys as watersheds and watercourses: slope lines that connect saddle points to local maxima or minima. Gauch and Pizer (1993) define ridges in terms of differential geometry and topography, and track them through multiple scales of image feature size. More generally, the localization of ridge and edge features in both position and intrinsic scale is a focus of extensive research (see e.g. Lindeberg (1998) or ter Haar Romeny (2003) and references therein). Most relevant for our approach, Eberly et al. (1994) motivate the idea that creases should be defined locally and be invariant with respect to a variety of transforms (rigid transforms, uniform scaling, and monotonic mappings of intensity), and they generalize the height-based definition of de Saint-Venant to $d$-dimensional manifolds embedded in $n$ dimensional image space. Other previous work focuses on extracting polygonal models of crease geometry; this is reviewed in Section 3.1.

We propose that a skeleton of the major white matter structures can be approximated from creases extracted directly from the differential properties of scalar-valued tensor attributes. Given the ubiquity of FA as a quantitative variable in the diffusion tensor literature, we start by detecting creases in FA, and term these anisotropy creases (Kindlmann et al., 2006). We propose that the ridge surfaces and ridge lines of FA coincide with the interiors of white matter fiber tracts, and that valley surfaces of anisotropy delineate the interfaces between fiber tracts that are adjacent but distinctly oriented (such as between the corpus callosum and the cingulum bundles). Anisotropy creases may have utility in a variety of contexts, such as non-rigid registration and shape analysis. The ability to extract white matter skeletons directly from tensor invariants, without the algorithmic complexity or parameter tuning of fiber tracking and clustering, could also increase sensitivity in shape analysis studies. We emphasize that our algorithm is fundamentally a local structural analysis of tensor image features, rather than a global connectivity analysis. That is, we seek geometric models of major white matter structures and their interfaces, not a detailed connectivity model over the entire brain.

Major crease features may also play a role analogous to that of the cortical surface in functional imaging, that is, a reference manifold onto which variables of interest are projected and analyzed. This general strategy is advanced by the Tract-Based Spatial Statistics (TBSS) method of Smith et al. (2006) (see Rouw and Scholte (2007) for an example application). TBSS enables voxel-based morphometry on a white matter skeleton calculated from ridges in a smooth mean FA image (from a set of registered scan), although "ridges" are not mentioned per se, and the ridge representation is a discrete raster image. By using an established mathematical definition of crease features, our technique extracts true codimension-one crease surfaces from continuous tensor fields, from individual DTI scans. Other examples of previous work in feature detection in DTI have also used, as we do, derivatives in tensor fields rather 
than tractography. Pajevic et al. (2002) use B-splines to generate continuous tensor fields that are differentiated to highlight anisotropy boundaries. O'Donnell et al. (2004) use structure tensors to detect general boundaries in tensor values. In both cases, results are visually evaluated by confirming a high edge strength near structural boundaries, but the techniques do not analyze the familiar FA measure, nor is the feature geometry explicitly extracted.

\section{Theoretical Background}

\subsection{Fractional Anisotropy and its Derivatives}

Our method is based on measuring the differential structure of fractional anisotropy (FA), so we review here the definition of FA (which leads to formulae for its spatial derivatives) and a method of creating a second-order continuous tensor field from sampled data. We notate the tensor trace and determinant as $\operatorname{tr}()$ and $\operatorname{det}()$, respectively, the identity tensor as $\mathbf{I}$, and the tensor norm as $|\mathbf{D}|=\sqrt{\operatorname{tr}\left(\mathbf{D D}^{T}\right)}$. The eigenvalues $\lambda_{\mathrm{i}}$ of tensor $\mathbf{D}$ are the roots of the characteristic polynomial $p(\lambda)=\operatorname{det} \lambda \mathbf{I}-\mathbf{D}$, and the coefficients of $p(\lambda)$ are the principal invariants $J_{1}, J_{2}, J_{3}$ (Bourne and Kendall, 1977). An additional invariant $J_{4}$ can be defined for convenience:

$J_{1}=\operatorname{tr}(\mathbf{D}) ; \quad J_{2}=\frac{\operatorname{tr}(\mathbf{D})^{2}-\operatorname{tr}\left(\mathbf{D}^{2}\right)}{2} ; \quad J_{3}=\operatorname{det}(\mathbf{D}) ; \quad J_{4}=|\mathbf{D}|^{2}$.

Basser and Pierpaoli (1996) define FA in terms of the deviatoric tensor $\tilde{\mathbf{D}}=\mathbf{D}-\operatorname{tr}(\mathbf{D}) \mathbf{I} / 3$ and tensor contraction $\mathbf{A}: \mathbf{B}=\operatorname{tr}\left(\mathbf{A B}^{\mathrm{T}}\right)$, which with some algebra can be re-expressed in terms of $J_{2}$ and $J_{4}$ :

$$
\mathrm{FA}=\sqrt{\frac{3}{2} \frac{\tilde{\mathbf{D}: \mathbf{D}}}{\mathbf{D}: \mathbf{D}}}=\sqrt{1-J_{2} / J_{4}} .
$$

$J_{2}$ and $J_{4}$ can in turn be expressed in terms of the individual tensor coefficients:

$$
\begin{gathered}
J_{2}=D_{x x} D_{y y}+D_{x x} D_{z z}+D_{y y} D_{z z}-D_{x y}^{2}-D_{x z}^{2}-D_{y z}^{2} \\
J_{4}=D_{x x}^{2}+D_{y y}^{2}+D_{z z}^{2}+2 D_{x y}^{2}+2 D_{x z}^{2}+2 D_{y z}^{2}
\end{gathered}
$$

Applying the chain rule to Equation 2 generates an expression for the spatial gradient of FA in terms of the spatial gradients of the individual tensor components:

$$
\begin{gathered}
\nabla \mathrm{FA}=\frac{J_{2} \nabla J_{4}-J_{4} \nabla J_{2}}{2 J_{4}^{2} \mathrm{FA}} \\
\nabla J_{2}=\left(D_{y y}+D_{z z}\right) \nabla D_{x x}+\left(D_{x x}+D_{z z}\right) \nabla D_{y y}+\left(D_{x x}+D_{y y}\right) \nabla D_{z z} \\
-2\left(D_{x y} \nabla D_{x y}+D_{x z} \nabla D_{x z}+D_{y z} \nabla D_{y z}\right) \\
\nabla J_{4}=2\left(D_{x x} \nabla D_{x x}+D_{y y} \nabla D_{y y}+D_{z z} \nabla D_{z z}\right) \\
+4\left(D_{x y} \nabla D_{x y}+D_{x z} \nabla D_{x z}+D_{y z} \nabla D_{y z}\right)
\end{gathered}
$$

The formula for the second derivative (the Hessian) of FA is given in Appendix A. Our formulaic decomposition of the derivatives of FA also translates to an implementation strategy, in which $J_{2}, J_{4}$ and their spatial derivatives are numerically computed (as described in the next section), and then combined to find the gradient and Hessian of FA. 


\subsection{Continuous Field Reconstruction}

Our method of crease extraction relies on a second-order continuous $\left(C^{2}\right)$ reconstruction of the tensor field from the discretely sampled data. Previous work has advanced the use of cubic Bsplines for interpolating the sampled tensor coefficients to create a $C^{2}$ tensor field (Aldroubi and Basser, 1999; Pajevic et al., 2002). The geometric structure of the crease features, however, does not depend on exact interpolation of the tensor values, and for the purposes of robustness with respect to sample noise it is advantageous for some amount of blurring to be incorporated into the field reconstruction. More generally, techniques in computer vision for localizing image features in scale-space typically use Gaussian blurring to low-pass filter the image data (Lindeberg, 1998; ter Haar Romeny, 2003).

We have as yet not pursued a full scale-space extraction of anisotropy creases, but we have used Gaussian blurring at a fixed scale as a pre-process (details in Section 4), followed by separable reconstruction with the uniform cubic B-spline function $b(x)$ as a (non-interpolating) $C^{2}$ kernel.

$$
b(x)= \begin{cases}0 & |x|>2 \\ -(|x|-2)^{3} / 6 & 1<|x|<2 \\ (|x|-2)|x|^{2} / 2+2 / 3 & 0<|x|<1\end{cases}
$$

By linearity of convolution-based reconstruction and differentiation, analytic derivatives of the reconstructed field are measured by convolving the sampled data with derivatives of the reconstruction kernel (Gonzalez and Woods, 2002). The partial derivatives of the separable three-dimensional reconstruction kernel $B(x, y, z)=b(x) b(y) b(z)$ can be determined by, for example, $\partial B / \partial x=b^{\prime}(x) b(y) b(z)$. The uniform cubic B-spline kernel $b(x)$ does not "ring" or add additional extrema in the reconstructed function, which in our experiments has avoided the creation of false crease responses around tissue boundaries.

As should be clear from Equation 2, FA is a nonlinear function of the tensor: $\mathrm{FA}(\mathbf{A}+\mathbf{B}) \neq \mathrm{FA}$ (A) + FA(B). This has bearing on how FA and its spatial derivatives are computed from discretely sampled tensor data. Specifically, pre-computing FA on a discrete grid and then convolving or differentiating (an approach taken by, for example, Goodlett et al. (2006)) is not equivalent to analytically calculating the FA and its derivatives (by Equation 5) of a continuous tensor field reconstruction. Figure 1 demonstrates this with a two-dimensional coronal slice of a human brain DTI scan (acquisition details given in Section 4). One of the contributions of our approach is incorporating the analytic calculation of FA (and its derivatives) directly into the crease-finding algorithm, rather than the more straight-forward approach of pre-computing a scalar FA field and then applying an existing crease-finding method.

\subsection{Crease Feature Definition}

Eberly et al. (1994) define crease features of a scalar field $f$ in terms of the gradient $\mathbf{g}=\nabla f$ and Hessian $\mathbf{H}$, in a way that easily generalizes to three and higher-dimensional images. Intuitively, creases are loci of constrained extrema, with the constraint surface defined by tangency to one or more Hessian eigenvectors. A function is at extrema where its gradient is orthogonal to the constraint surface (Marsden and Tromba, 1996), so in three dimensions ridges and valleys occur where the gradient $\mathbf{g}$ is orthogonal to one or two of the unit-length eigenvectors $\left\{\mathbf{e}_{1}, \mathbf{e}_{2}\right.$, $\mathbf{e}_{3}$ \} (with corresponding sorted eigenvalues $\lambda_{1} \geq \lambda_{2} \geq \lambda_{3}$ ) of the Hessian $\mathbf{H}$ :

In this definition, the strength of the image feature can be assessed by the magnitude of the eigenvalues that are required to be negative or positive for ridges and valleys, respectively, 
which can be used to filter out insignificant features. Specifically, crease surface strength is measured by $-\lambda_{3}$ (for ridges) and $\lambda_{1}$ (for valleys), and crease line strength is measured by $-\lambda_{2}$ (for ridges) and $\lambda_{2}$ (for valleys).

\section{Methods}

\subsection{Crease Surface Extraction}

In our work to date we have implemented geometric extraction of crease surfaces, but not crease lines, because surfaces constitute the majority of the fiber tract geometry that we seek to capture. We extract crease surfaces by per-voxel triangulation of the zero-isocontour of $\mathbf{g}$. $\mathbf{e}_{i}\left(\mathbf{g} \cdot \mathbf{e}_{3}\right.$ for ridges, $\mathbf{g} \cdot \mathbf{e}_{1}$ for valleys) using Marching Cubes (MC) (Lorensen and Cline, 1987). Straight-forward application of MC, however, would not produce useful results, because the inherent sign ambiguity of eigenvectors leads to arbitrary signs ing $\cdot \mathbf{e}_{i}$, which must be consistent for the MC case tables to apply. The literature offers ways to overcome this. Morse (1994) suggests determining correspondences between sets of eigenvectors rather than individual ones, to handle eigenvector permutations associated with eigenvalue equality. Furst et al. (1996) use similar ideas in Marching Cores to extract crease manifolds in image scalespace. In their Marching Ridges method, Furst and Pizer (2001) compute the principal eigenvector of the average of outer products of the eigenvectors at voxel corners, and use its (arbitrary) sign to impose a consistent sign to eigenvectors at voxel corners.

We have taken a more cautious approach, based on observations suggesting that eigenvectors of the Hessian of non-linear scalar attributes of tensors (such as FA) can vary more rapidly than Hessian eigenvectors of a similarly sampled scalar field, which may confound the heuristics (described above) developed for scalar fields. Thus, we determine the correspondence between eigenvector signs at the voxel corners by continuously tracking the eigenvector orientation along each voxel edge. Point samples are adaptively generated along all voxel edges until the angle between eigenvectors at adjacent samples are reduced below a threshold (20 degrees in our current work). This process, which is currently the most timeconsuming component of our approach, determines whether the smooth transport of $\mathbf{e}_{i}\left(\mathbf{v}_{0}\right)$ from vertex $\mathbf{v}_{0}$ to vertex $\mathbf{v}_{1}$ agrees in sign with eigenvector $\mathbf{e}_{i}\left(\mathbf{v}_{1}\right)$ or $-\mathbf{e}_{i}\left(\mathbf{v}_{1}\right)$ computed at $\mathbf{v}_{1}$. The peredge eigenvector sign information is propagated along all voxel edges to determine a pervoxel sign consistency prior to evaluating the MC case. This process ensures intravoxel eigenvector sign consistency, but not inter-voxel sign consistency, which creates triangulations with inconsistent vertex windings (the clockwise versus counter-clockwise order in which the triangle's vertices are listed). Thus, as a final pass, we traverse the crease surface mesh to make the vertex windings consistent, while splitting the surface with vertex duplication in the case of non-orientable surfaces. This allows graphics hardware to appropriately render the crease surfaces with two-sided lighting (Shreiner et al., 2004).

The remaining details of our method can decrease the computation time and increase the utility of the results. We refer to the grid on which the crease surface is triangulated one voxel at a time (by our modified MC) as the triangulation grid. The continuous tensor field measurements (Section 2.2) allow the resolution of the triangulation grid to be independent of the sampling of underlying tensor data. A triangulation grid with finer sampling (higher resolution) than the underlying data improves the quality of the crease surface triangulation, yet no up-sampling to the triangulation grid is required as a pre-process. By sampling the crease surface strength (FA Hessian eigenvalue $-\lambda_{3}$ for ridges, $\lambda_{1}$ for valleys) at the vertices of triangulation grid, we can skip the voxels that are unlikely to contribute to the crease surface. Even with these precautions, the extracted crease surfaces are often made of many disjoint pieces. We use connected-component analysis on the polygonal crease surfaces to filter out all but the largest few of the crease surface components. We believe that removing the smallest components increases the anatomic significance of the crease surface results by removing the pieces that 
are most apt to vary as a function of image noise and scale selection. This geometric postprocessing is a significant improvement over the method presented in our previous work (Kindlmann et al., 2006).

We demonstrate the method on a synthetic dataset prior to giving results on DTI scans. Figure 2 shows results on a synthetic dataset containing two adjacent bands of orthogonally oriented linear anisotropy. The ridge surfaces of FA represent the interiors of the two bands, and the valley surface of FA marks the interface between them. It is in this sense that we claim that crease surfaces model the skeleton of the white matter fiber geometry.

\section{Results}

The results in this section used tensor data acquired on a $1.5 \mathrm{~T}$ Philips scanner with SENSE parallel imaging (reduction factor 2.5), using single-shot spin echo EPI diffusion-weighted images along 30 non-collinear gradient directions $\left(b=700 \mathrm{~s} / \mathrm{mm}^{2}\right)$, with five non-diffusionweighted T2 images. The field of view, the size of the acquisition matrix, and the slice thickness were $240 \times 240 \mathrm{~mm}, 96 \times 96$, and $2.5 \mathrm{~mm}$, respectively, leading to an image resolution of $2.5 \mathrm{~mm}^{2} .55$ axial slices were acquired to cover the entire hemisphere and the cerebellum. Unless otherwise noted, the standard deviation $\sigma$ of the Gaussian smoothing kernel (prior to B-spline-based reconstruction and differentiation) was $1.25 \mathrm{~mm}$, and the resolution of the triangulation grid is five times that of the underlying tensor dataset. The computation timings are with a $1.67 \mathrm{GHz}$ PowerPC G4 with 2 GB of memory.

\subsection{Slice Inspection}

Prior to their geometric extraction, the numerical ingredients of anisotropy crease features can be visualized on densely sampled slices. Figure 3 shows the numerical constituents of crease features on the same dataset slice used in Fig. 1. The strength measures described in Section 2.3 are visualized in the top half of the figure. These are used to modulate the display in the bottom half of the figure of the scalar function whose zero-level defines the crease feature, with some additional contrast enhancement for clarity. Crease surfaces intersect with the cutting plane in curves, and crease lines intersect with the plane in points. Note that the ridge surfaces (Fig. 3(d)) closely follow the shape of the major white matter tracts (annotated in Fig. 4(a)), and the valley surfaces (Fig. 3(e)) delineate the interface between tracts that are adjacent but distinctly oriented. The anatomic utility of the ridge lines (Fig. 3(f)) is limited to tracts with a more cylindrical shape, that is, features for which the two smallest eigenvalues $\lambda_{2}$ and $\lambda_{3}$ of the Hessian are approximately equal. This includes the cingulum bundles and fornix (Mori et al., 2005), although the non-zero response of $s_{r l}$ through-out the slice complicates visual confirmation.

The renderings in Fig. 4 (from a posterior viewpoint) show a cropped region around the same coronal slice of previous figures. In Fig. 4(a) fibers are seeded from the RGB encoded plane, and some of the major pathways are indicated. Fig. 4(b) shows how the ridge surfaces (using the same RGB encoding) follow major fiber paths, especially the corpus callosum (CC), internal capsule (IC), corona radiata (CR), and fornix (FX) (Mori et al., 2005). The (white) anisotropy valley surfaces in Fig. 4(c) delineate interfaces between the $\mathrm{CC}$ and cingulum bundles (CB), superior fronto-occipital fasciculus (SFO) and IC, and IC and superior longitudinal fasciculus (SLF). Figure 4(d) also illustrates how anisotropy valleys lie between adjacent paths of differing orientation. The ridge and valley surface extractions each took 10 minutes.

Figure 5 illustrates anisotropy crease analysis in the brainstem (lateral anterior superior viewpoint), a region with a closely contained complex of fiber pathways along distinct directions (Salamon et al., 2005). For anatomical context, Fig. 5(a) shows fiber tractography 
results, including the (from ventral to dorsal) middle cerebellar peduncle (MCP), corticospinal tract (CST), transverse pontine tract (TPT), medial lemniscus (ML), superior cerebellar peduncle (SCP), and inferior cerebellar peduncle (ICP). These pathways appear as anisotropy ridge surfaces in Fig. 5(b). The tract interfaces are delineated by the valley surfaces in Fig. 5 (c), which also includes for reference a translucent cutting plane with RGB coloring of the principal diffusivity direction, as well as a view-aligned clipping plane to reveal the RGB color differences among the MCP, CST, and TPT. Figure 5(d) illustrates how valley surfaces delineate major fiber bundles, faintly visible as tractography results. In particular, valley surface patches are visible between MCP and CST, CST and TPT, TPT and ML, MCP and ICP, and ICP and SCP, all of which are locations where distinctly oriented fiber pathways pass near each other. The ridge and valley surface extractions each took 9 minutes.

Figure 6 demonstrates the connected-component analysis mentioned in Section 3.1, which allows the main crease surface components to be extracted from the much larger collection of disjoint pieces. For these results and those in Fig. 7, the tensor datasets were down-sampled by a factor of two, to simplify the field to only its largest features. Ridge surface extraction on this dataset produced 742 surface connected components (CCs), but Fig. 6 shows that the largest nine CCs capture nearly all the large fiber tracts visible in all CCs.

To demonstrate the repeatability of our method, Fig. 7 expands the results from Fig. 6 to include five other scans from the same database, all processed identically. The computation of these ridge surfaces took 6 minutes per dataset. In every case, the single largest connected component contains a single surface representing the corpus callosum, as well as parts of other pathways, such as the corona radiata, and often the internal capsules. We are currently investigating why ridge surface patches corresponding to distinct fiber pathways can appear joined in our ridge surface analysis, which is likely related to the problem of kissing or crossing fiber tracts.

\section{Discussion and Future Work}

The preliminary results in this work suggest that anisotropy creases delineate the major white matter structure in DTI, and that analytically measured anisotropy derivatives (and an adaptation of Marching Cubes) can lead to high-quality triangulated models of crease surface geometry. In contrast to the combination of tractography and clustering algorithms, the invariance properties in the mathematical definition of anisotropy creases give them the attractive property of having very few parameters in their extraction. The main parameter is the scale of the Gaussian smoothing pre-process, since this puts a lower bound on the scale of the extracted features. Secondary parameters include the feature strength threshold (Section 2.3), the resolution of the crease triangulation grid, and the manner of selecting connectedcomponents from the triangulation output (Section 3).

As mentioned in Section 1, our method is a structural analysis of features in DTI, and not the connectivity analysis that is a popular research focus. This has implications for the type of studies for which anisotropy creases may be applied in future work (described below), as our focus is on the over-all shape of the white matter pathways, rather than on their course. In particular, for the task of extracting a geometric model of the major white matter pathways, we accept simplicity of the single tensor model, despite its well-known inability to represent fiber crossings and multiple fiber orientations (Alexander et al., 2002; Tuch et al., 2002), because it gives rise to a tractable analysis of the differential properties of anisotropy as parameterized by FA (Eq. 5), and because for large portions of the major pathways (e.g., visualized in Fig. 4(b)), the single tensor model appears sufficient. Indeed, for the extraction of fiber pathway interfaces (Fig. 4(c)), we embrace the fact that the partial voluming of distinctly oriented pathways gives rise to artifactual planar anisotropy by component-wise interpolation (Alexander et al., 2001), which leads to lower FA values (Fig. 2(b)), which in 
turn enables FA valley surfaces to geometrically isolate the interfaces (Figs. 2(c) and 4(c)). On the other hand, for future work one could also consider measuring the differential properties of continuous maps of non-tensor or higher-order anisotropy measures (Özarslan et al., 2005; Descoteaux et al., 2006), which could possibly generate geometric models of areas of fibercrossings in particular, or highlight where our current FA-based analysis could be most misleading.

The continuous tensor field reconstruction method we use (Aldroubi and Basser, 1999; Pajevic et al., 2002) is "Euclidean" in that it implicitly considers diffusion tensors as elements of a vector space, even though this neglects the strictly positive-definite nature of diffusion. The DTI literature provides a precedent for this simplifying assumption in other contexts as well (Basser et al., 2000; Basser and Pajavic, 2003; Basser and Pajevic, 2007). In a different line of recent work, however, tensors are located on a Riemannian manifold that effectively creates an infinite distance between valid tensors and those with zero determinant (Pennec, 2004; Fletcher and Joshi, 2004; Batchelor et al., 2005; Lenglet et al., 2006), and the Log-Euclidean methods provide computationally efficient approximations (Arsigny et al., 2006; Fillard et al., 2006). The relative strengths of these two approaches (Euclidean versus Riemannian) is an ongoing issue that we do not intend to resolve here, although some basic points bear consideration. First, it is possible to define tensor field derivatives on a Riemannian manifold (Lenglet et al., 2006), from which anisotropy derivatives could also be computed. Thus, in principal anisotropy creases could be extracted entirely within a Riemannian framework, though at an increased computation cost. On the other hand, the distinction between the Euclidean and Riemannian approaches may be viewed more simply as a difference in choosing whether to enforce positive-definiteness solely at the data acquisition stage (Euclidean), or also at the analysis stage (Riemannian). We view the enforcement of this constraint as orthogonal to the task of analyzing the differential structure of anisotropy, especially since FA is defined without regard to positive-definiteness (which has apparently not hindered its scientific utility).

Our ongoing algorithmic work is focused on geometric extraction of FA ridge lines (for example through the cingulum bundles), and then comparing the FA ridge lines to individual fiber tracts. There are also theoretical questions remaining about the differential structure of FA. Given the scale of the Gaussian smoothing pre-process, and the properties of the cubic reconstruction kernel, we would like to determine an upper bound on the resolution of the surface triangulation grid (relative to the original tensor samples) that is guaranteed to find all anisotropy creases. In the case of valleys in FA, we would like to quantify the relationship between strength and location of the valley surface, and the linear anisotropy levels and orientation of the two neighboring regions.

Developing applications of anisotropy crease features is the main focus of future work. Following the example of TBSS (Smith et al., 2006; Rouw and Scholte, 2007), we are interested in using anisotropy crease features as manifolds onto which neuroanatomic variables can be projected for the purposes of comparison and study. While TBSS generates a discretized FA ridge surface map, we are curious whether having true codimension-one surfaces, for both ridges and valleys, extracted from individual cases instead of a group registration result, can increase the statistical power of analysis based on anisotropy creases. The second main application area for future work is in providing fiducial markers to drive non-rigid registration of DTI. Related work is described in Goodlett et al. (2006), although here too the results are in the form of discrete images rather than continuous surfaces. Using valley surfaces of FA to explicitly model the interfaces between adjacent but orthogonal fiber tracts may usefully guide non-rigid registration of tensor fields for group studies. Slight mis-registration of these configurations could lead to comparison of tensor values within entirely separate pathways. In addition, just as shape analysis of segmented structures from scalar MRI is an active area of 
medical image research (e.g., Styner et al. (2004)), anisotropy creases from DTI may also also support informative shape analysis.

Finally, to extract true image cores, crease detection must work simultaneously across image scales (Furst et al., 1996; Lindeberg, 1998). This will incur greater computational cost, and perhaps algorithmic complexity, but we are investigating methods to limit the computation to regions around crease features (generalizing the existing strategy of skipping voxels with low feature strength). We believe that incorporating scale space analysis into our crease feature extraction may give the method better noise robustness, because feature strength would be stronger at the larger scales where noise is smoothed out.

\section{Acknowledgments}

This work supported by NIH grants NIBIB T32-EB002177, U41 RR019703 (IGT), NCRR P41-RR13218 (NAC) and P41-RR12553-07 (CIBC), R01-MH050740, and R01-MH074794. DWI data courtesy of Dr. Susumu Mori, Johns Hopkins University, supported by NIH R01-AG20012-01 and P41-RR15241-01A1

\section{A Hessian of Fractional Anisotropy}

The Hessian of fractional anisotropy (FA) is determined by differentiating Equations 5, 6, and 7. The differential operator $\nabla$ produces a column vector, and $\mathbf{H}=\nabla \nabla^{\mathrm{T}}$ produces the Hessian matrix of second partial derivatives.

$$
\begin{aligned}
\mathbf{H}(\mathrm{FA})= & \frac{\nabla J_{2} \nabla^{\mathrm{T}} J_{4}+\nabla J_{4} \nabla^{\mathrm{T}} J_{2}+J_{2} \mathbf{H}\left(J_{4}\right)+J_{4} \mathbf{H}\left(J_{2}\right)}{2 J_{4}^{2} \mathrm{FA}} \\
& -\frac{\left(2 J_{4} \nabla J_{4} \mathrm{FA}+J_{4}^{2} \nabla \mathrm{FA}\right)\left(J_{2} \nabla^{\mathrm{T}} J_{4}-J_{4} \nabla^{\mathrm{T}} J_{2}\right)}{2\left(J_{4}^{2} \mathrm{FA}\right)^{2}}
\end{aligned}
$$

$$
\begin{aligned}
\mathbf{H}\left(J_{2}\right) & =\left(\nabla D_{y y}+\nabla D_{z z}\right) \nabla^{\mathrm{T}} D_{x x}+\left(D_{y y}+D_{z z}\right) \mathbf{H}\left(D_{x x}\right) \\
& +\left(\nabla D_{x x}+\nabla D_{z z}\right) \nabla^{\mathrm{T}} D_{y y}+\left(D_{x x}+D_{z z}\right) \mathbf{H}\left(D_{y y}\right) \\
& +\left(\nabla D_{x x}+\nabla D_{y y}\right) \nabla^{\mathrm{T}} D_{z z}+\left(D_{x x}+D_{y y}\right) \mathbf{H}\left(D_{z z}\right) \\
& -2\left(\nabla D_{x y} \nabla^{\mathrm{T}} D_{x y}+D_{x y} \mathbf{H}\left(D_{x y}\right)+\nabla D_{x z} \nabla^{\mathrm{T}} D_{x z}+D_{x z} \mathbf{H}\left(D_{x z}\right)+\nabla D_{y z} \nabla^{\mathrm{T}} D_{y z}+D_{y z} \mathbf{H}\left(D_{y z}\right)\right)
\end{aligned}
$$

$$
\begin{aligned}
\mathbf{H}\left(J_{4}\right) & =2\left(\nabla D_{x x} \nabla^{\mathrm{T}} D_{x x}+D_{x x} \mathbf{H}\left(D_{x x}\right)+\nabla D_{y y} \nabla^{\mathrm{T}} D_{y y}+D_{y y} \mathbf{H}\left(D_{y y}\right)+\nabla D_{z z} \nabla^{\mathrm{T}} D_{z z}+D_{z z} \mathbf{H}\left(D_{z z}\right)\right) \\
& +4\left(\nabla D_{x y} \nabla^{\mathrm{T}} D_{x y}+D_{x y} \mathbf{H}\left(D_{x y}\right)+\nabla D_{x z} \nabla^{\mathrm{T}} D_{x z}+D_{x z} \mathbf{H}\left(D_{x z}\right)+\nabla D_{y z} \nabla^{\mathrm{T}} D_{y z}+D_{y z} \mathbf{H}\left(D_{y z}\right)\right)
\end{aligned}
$$

\section{References}

Aldroubi A, Basser P. Reconstruction of vector and tensor fields from sampled discrete data. Contemporary Mathematics 1999;247:1-15.

Alexander A, Hasan K, Lazar M, Tsuruda J, Parker D. Analysis of partial volume effects in diffusiontensor MRI. Magnetic Resonance in Medicine 2001;45:770-780. [PubMed: 11323803]

Alexander D, Barker G, Arridge S. Detection and modeling of non-gaussian apparent diffusion coefficients profiles in human brain data. Magnetic Resonance in Medicine 2002;48:331-340. [PubMed: 12210942]

Arsigny V, Fillard P, Pennec X, Ayache N. Log-Euclidean metrics for fast and simple calculus on diffusion tensors. Magnetic Resonance in Medicine August;2006 56(2):411-421. [PubMed: 16788917]

Basser P. Inferring microstructural features and the physiological state of tissues from diffusion-weighted images. Nuclear Magnetic Resonance in Biomedicine 1995;8:333-344.

Basser P, Jones D. Diffusion-tensor MRI: Theory, experimental design and data analysis - A technical review. Nuclear Magnetic Resonance in Biomedicine 2002;15:456-467. 
Basser P, Mattiello J, LeBihan D. MR diffusion tensor spectroscopy and imaging. Biophysics Journal 1994;66(1):259-267.

Basser PJ, Pajavic S. A normal distribution for tensor-valued random variables: Applications to diffusion tensor MRI. IEEE Transactions on Medical Imaging July;2003 22(7):785-794. [PubMed: 12906233]

Basser PJ, Pajevic S. Spectral decomposition of a 4th-order covariance tensor: Applications to diffusion tensor MRI. Signal Processing 2007;87:220-236.

Basser PJ, Pajevic S, Pierpaoli C, Duda J, Aldroubi A. In vivo fiber tractograpy using DT-MRI data. Magnetic Resonance in Medicine 2000;44:625-632. [PubMed: 11025519]

Basser PJ, Pierpaoli C. Microstructural and physiological features of tissues elucidated by quantitativediffusion-tensor MRI. Journal of Magnetic Resonance 1996;111:209-219. [PubMed: 8661285] Series B

Batchelor PG, Moakher M, Atkinson D, Calamante F, Connelly A. A rigorous framework for diffusion tensor calculus. Magnetic Resonance in Medicine January;2005 53(1):221-225. [PubMed: 15690523]

Beaulieu C. The basis of anisotropic water diffusion in the nervous system - A technical review. Nuclear Magnetic Resonance in Biomedicine 2002;15:435-455.

Bourne, D.; Kendall, P. Vector Analysis and Cartesian Tensors. 2nd Edition. Thomas Nelson and Sons, Ltd; Great Britain: 1977. Ch. 9

Corouge I, Fletcher P, Joshi S, Gouttard S, Gerig G. Fiber tract-oriented statistics for quantitative diffusion tensor MRI analysis. Medical Image Analysis 2006;10(5):786-798. [PubMed: 16926104]

de Saint-Venant M. Surfaces à plus grande pente constituées sur des lignes courbes. Bulletin de la Société Philomathématique de Paris 1852:24-30.

Descoteaux M, Angelino E, Fitzgibbons S, Deriche R. Apparent diffusion coefficients from high angular resolution diffusion imaging: Estimation and applications. Magnetic Resonance in Medicine 2006;56:395-410. [PubMed: 16802316]

Dougherty RF, Ben-Shachar M, Deutsch G, Potanina P, Bammer R, Wandell BA. Occipital-Callosal pathways in children: Validation and atlas development. Annals of the New York Academy of Sciences 2005;1064:98-112. [PubMed: 16394151]

Eberly D, Gardner R, Morse B, Pizer S. Ridges for image analysis. Journal of Mathematical Imaging and Vision 1994;4:351-371.

Fillard, P.; Arsigny, V.; Pennec, X.; Ayache, N. Clinical DT-MRI estimation, smoothing and fiber tracking with Log-Euclidean metrics. Proceedings of the Third IEEE International Symposium on Biomedical Imaging (ISBI 2006); LNCS; Arlington, Virginia, USA. Apr. 2006 p. 786-789.

Fletcher, PT.; Joshi, S. Proceedings ECCV 2004 Workshop on Computer Vision Approaches to Medical Image Analysis (CVAMIA). 31107 of LNCS. Springer-Verlag; 2004. Principal geodesic analysis on symmetric spaces: Statistics of diffusion tensors; p. 87-98.

Furst, JD.; Pizer, SM. Marching ridges; Proceedings of 2001 IASTED International Conference on Signal and Image Processing; 2001. p. 22-26.

Furst, JD.; Pizer, SM.; Eberly, DH. Marching cores: A method for extracting cores from 3D medical images; Proceedings of IEEE Workshop on Mathematical Methods in Biomedical Image Analysis; Jun. 1996 p. 124-130.

Gauch JM, Pizer SM. Multiresolution analysis of ridges and valleys in grey-scale images. IEEE Transactions on Pattern Analysis and Machine Intelligence June;1993 15(6):635-646.

Gonzalez, R.; Woods, R. Digital Image Processing. 2nd Edition. Addison-Wesley Publishing Company; Reading, MA: 2002.

Goodlett, C.; Davis, B.; Jean, R.; Gilmore, JH.; Gerig, G. Improved correspondence for DTI population studies via unbiased atlas building. Ninth International Conference on Medical Image Computing and Computer-Assisted Intervention (MICCAI'06); Lecture Notes in Computer Science 4190; Copenhagen, Denmark. Oct. 2006 p. 260-267.

Haralick RM. Ridges and valleys on digital images. Computer Vision, Graphics, and Image Processing 1983;22:28-38.

Kanaan RAA, Kim J-S, Kaufmann WE, Pearlson GD, Barker GJ, McGuire PK. Diffusion tensor imaging in schizophrenia. Biological Psychiatry December;2005 58:921-929. [PubMed: 16043134] 
Kindlmann, G.; Tricoche, X.; Westin, C-F. Proceedings MICCAI 2006. Lecture Notes in Computer Science 4190; Copenhagen, Denmark: Oct. 2006 Anisotropy creases delineate white matter structure in diffusion tensor MRI; p. 126-133.

Klingberg T, Hedehus M, Temple E, Salz T, Gabrielli JDE, Moseley ME, Poldrack RA. Microstructure of temporo-parietal white matter as a basis for reading ability: Evidence from diffusion tensor magnetic resonance imaging. Neuron 2000;25:493-500. [PubMed: 10719902]

Kubicki M, Westin C-F, Maier S, Mamata H, Frumin M, Ernst-Hirshefeld H, Kikinis R, Jolesz F, McCarley R, Shenton M. Cingulate fasciculus integrity disruption in schizophrenia: A magnetic resonance diffusion tensor imaging study. Biological Psychiatry 2003;54:1171-1180. [PubMed: 14643084]

Lenglet C, Rousson M, Deriche R. DTI segmentation by statistical surface evolution. IEEE Transactions on Medical Imaging June;2006 25:685-700. [PubMed: 16768234]

Lindeberg T. Edge detection and ridge detection with automatic scale selection. International Journal of Computer Vision November;1998 30(2):77-116.

Lorensen WE, Cline HE. Marching cubes: A high resolution 3D surface construction algorithm. Computer Graphics 1987;21(4):163-169.

Marsden, J.; Tromba, A. Vector Calculus. W.H. Freeman and Company; New York, New York: 1996.

Maxwell J. On hills and dales. The London, Edinburgh and Dublin Philosophical Magazine and Journal of Science 1870;40(269):421-425.

Moberts B, Vilanova A, van Wijk JJ. Evaluation of fiber clustering methods for diffusion tensor imaging. Proceedings IEEE Visualization 2005 2005:65-72.

Mori, S.; Wakana, S.; Nagae-Poetscher, L.; Zijl, PV. MRI Atlas of Human White Matter; Elsevier: 2005.

Morse, BS. Computation of object cores from grey-level images. University of North Carolina at Chapel Hill; Chapel Hill, NC: 1994. Ph.D. thesis

O'Donnell L, Grimson W, Westin C-F. Interface detection in diffusion tensor MRI. Proceedings MICCAI 2004 2004:360-367.

O'Donnell L, Kubicki M, Shenton ME, Dreusicke M, Grimson WEL, Westin C-F. A method for clustering white matter fiber tracts. American Journal of Neuroradiology May;2006 27:1032-1036. [PubMed: 16687538]

Özarslan E, Vemuri BC, Mareci TH. Generalized scalar measures for diffusion mri using trace, variance, and entropy. Magnetic Resonance in Medicine 2005;53:866-876. [PubMed: 15799039]

Pajevic S, Aldroubi A, Basser P. A continuous tensor field approximation of discrete DT-MRI data for extracting microstructural and architectural features of tissue. Journal of Magnetic Resonance 2002;154:85-100. [PubMed: 11820830]

Pennec X. Probabilities and statistics on riemannian manifolds: A geometric approach. Tech. Rep. 5093, INRIA, Sophia Antipolis. January;2004

Pierpaoli C, Jezzard P, Basser P, Barnett A, DiChiro G. Diffusion tensor MR imaging of the human brain. Radiology Dec;1996 201(3):637-648. [PubMed: 8939209]

Rouw R, Scholte HS. Increased structural connectivity in grapheme-color synesthesia. Nature Neuroscience 2007;10:792-797.

Salamon N, Sicotte N, Alger J, Shattuck D, Perlman S, Sinha U, Schultze-Haakh H, Salamon G. Analysis of the brain-stem white-matter tracts with diffusion tensor imaging. Neuroradiology $2005 ; 47(12)$ : 895-902. [PubMed: 16158279]

Salat DH, Tuch DS, Hevelone ND, Fischl B, Corkin S, Rosas HD, Dale AM. Age-related changes in prefrontal white matter measured by diffusion tensor imaging. Annals of the New York Academy of Sciences 2005;1064:37-49. [PubMed: 16394146]

Shreiner, D.; Woo, M.; Neider, J.; Davis, T. OpenGL Programming Guide. 4th Edition. Addison-Wesley; 2004.

Smith SM, Jenkinson M, Johansen-Berg H, Rueckert D, Nichols TE, Mackay CE, Watkins KE, Ciccarelli O, Cader MZ, Matthews PM, Behrens TEJ. Tract-based spatial statistics: Voxelwise analysis of multisubject diffusion data. NeuroImage 2006;31:1487-1505. [PubMed: 16624579]

Styner M, Lieberman JA, Pantazis D, Gerig G. Boundary and medial shape analysis of the hippocampus in schizophrenia. Medical Image Analysis September;2004 8(3):197-203. [PubMed: 15450215] 
ter Haar Romeny, BM. Vol. 27 of Computational Imaging and Vision. Kluwer Academic Publishers; Dordrecht, The Netherlands: 2003. Front-End Vision and Multi-Scale Image Analysis.

Tuch DS, Reese TG, Wiegell MR, Makris N, Belliveau JW, Wedeen VJ. High angular resolution diffusion imaging reveals intravoxel white matter fiber heterogeneity. Magnetic Resonance in Medicine 2002;48:577-582. [PubMed: 12353272]

Tuch DS, Salat DH, Wisco JJ, Zaleta AK, Hevelone ND, Rosas HD. Choice reaction time performance correlates with diffusion anisotropy in white matter pathways supporting visuospatial attention. Proceedings of the National Academy of Sciences 2005;102(34):12212-12217.

Zhang S, Correia S, Tate DF, Laidlaw DH. Correlating DTI fiber clusters with white-matter anatomy. Proceedings International Society for Magnetic Resonance in Medicine (ISMRM) 2006:442. 


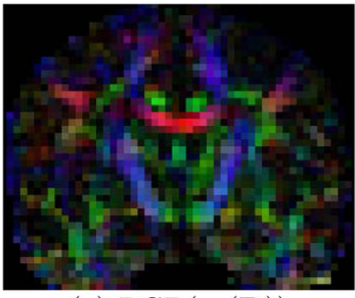

(a) $\operatorname{RGB}\left(\mathbf{e}_{1}(\mathbf{D})\right)$

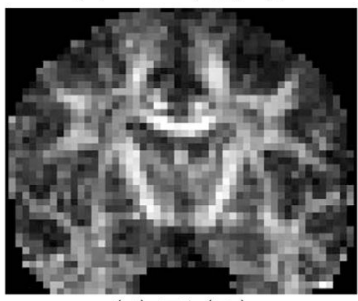

(d) FA(D)

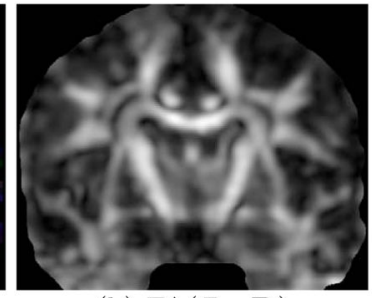

(b) $\mathrm{FA}(B \star \mathbf{D})$

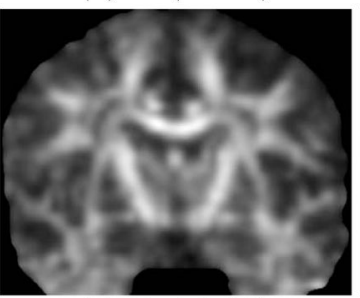

(e) $B \star \mathrm{FA}(\mathbf{D})$

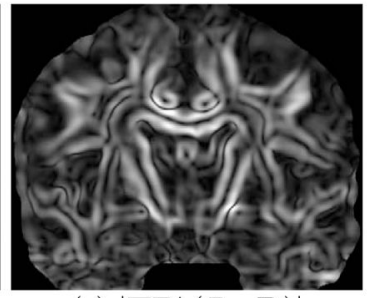

(c) $|\nabla \mathrm{FA}(B \star \mathbf{D})|$

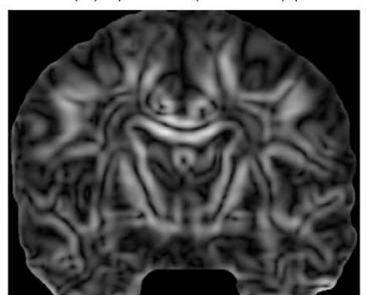

(f) $|\nabla B \star \mathrm{FA}(\mathbf{D})|$

Fig. 1.

FA calculation does not commute with convolution-based reconstruction or differentiation. A slice of sampled tensor data $\mathbf{D}$ is shown with the standard RGB colormap (a) (?) or FA map (d). Convolving the discrete tensor data with a continuous reconstruction kernel $B$ produces a continuous tensor field, in which we measure FA (b) and $|\nabla F A|(c)$. Convolving the discrete FA data FA(D) with $B$ gives a continuous FA field (e) and $|\nabla F A|$ (f). Note that the shape and boundaries of the finer structures in (b) appear blurred in (e), especially near the center of the image. This is confirmed by the $|\nabla F A|$ images; interfaces between major tracts visible in (a) remain sharp in (c) but indistinct in (f). 


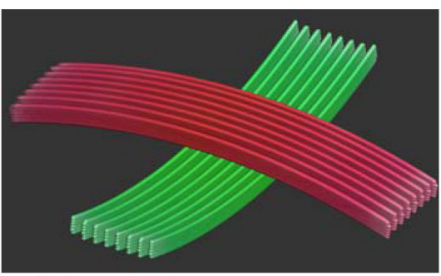

(a) Fiber tractography

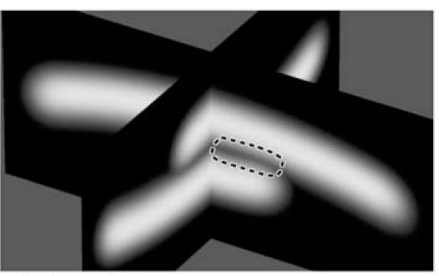

(b) FA on cutting planes

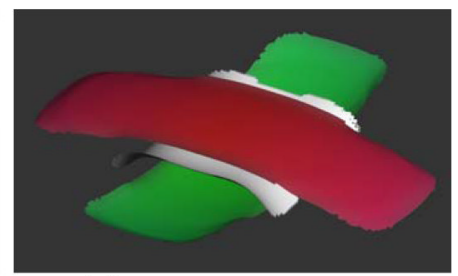

(c) Crease surfaces

Fig. 2.

Demonstrate of FA ridge and valley surfaces in a synthetic dataset. The tractography of the two $\operatorname{arcs}$ in (a) is color-coded by the usual $\operatorname{RGB}\left(\mathbf{e}_{1}\right)$. The lower FA between the arcs is highlighted in (b). In (c), the red and green ridge surfaces correctly model the shape of bands, and the white valley surface captures the interface between them. 


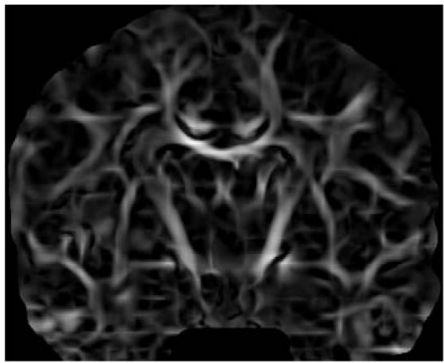

(a) $s_{r s}=\max \left(-\lambda_{3}, 0\right)$

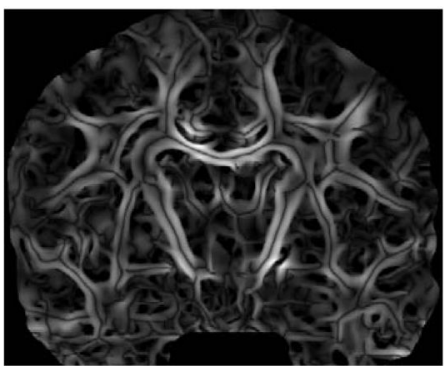

(d) $s_{r s}\left|\mathbf{g} \cdot \mathbf{e}_{3}\right|^{1 / 3}$

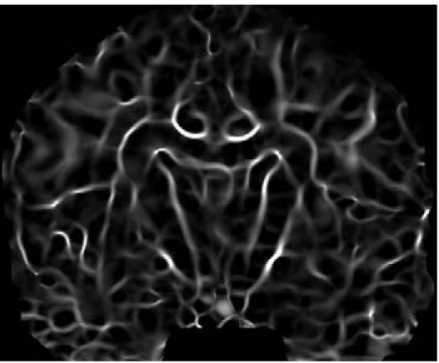

(b) $s_{v s}=\max \left(\lambda_{1}, 0\right)$

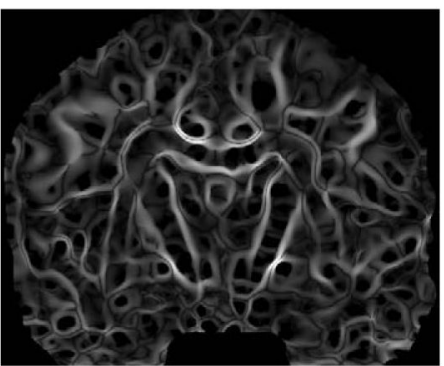

(e) $s_{v s}\left|\mathbf{g} \cdot \mathbf{e}_{1}\right|^{1 / 3}$

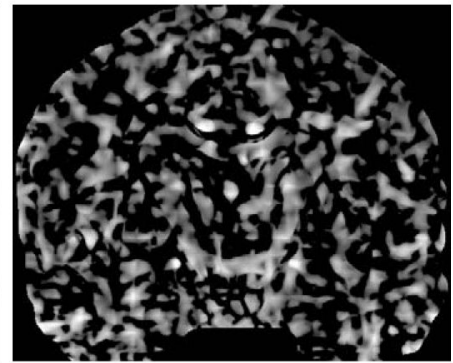

(c) $s_{r l}=\max \left(-\lambda_{2}, 0\right)$

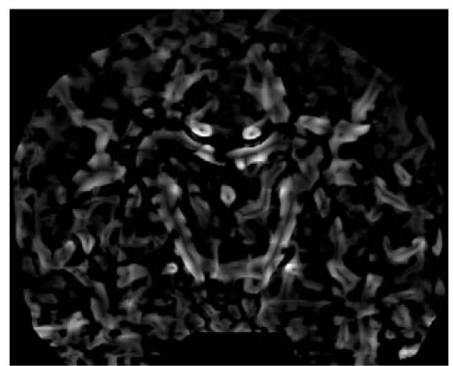

(f) $s_{r l} \sqrt{\left(\mathbf{g} \cdot \mathbf{e}_{2}\right)^{2}+\left(\mathbf{g} \cdot \mathbf{e}_{3}\right)^{2}}$

Fig. 3.

Functional components of crease feature definition. The ridge surface strength $s_{r s}(\mathrm{a})$, valley surface strength $s_{v s}$ (b), and ridge line strength $s_{r l}$ (c) are all defined in terms of the eigenvalues of the FA Hessian. These are used to modulate the display of the ridge surface (d), valley surface (e), and ridge line (f) functions defined in terms of the FA gradient $\mathbf{g}$ and Hessian eigenvectors $\mathbf{e}_{i}$. The crease features are visible as dark lines (in the case of crease surfaces) or dark dots (in the case of ridge lines) in the bright areas. 


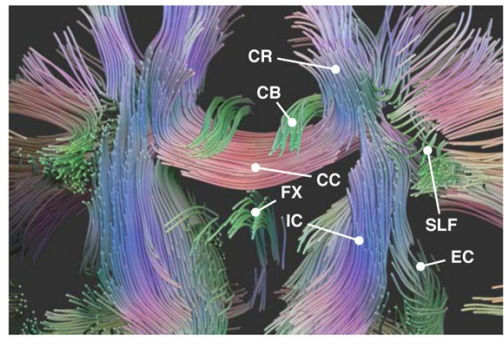

(a) RGB Map with Fibers

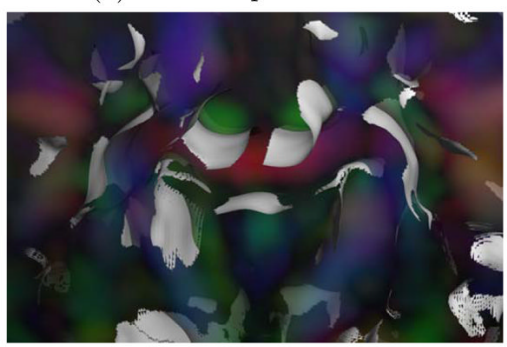

(c) Valley Surfaces

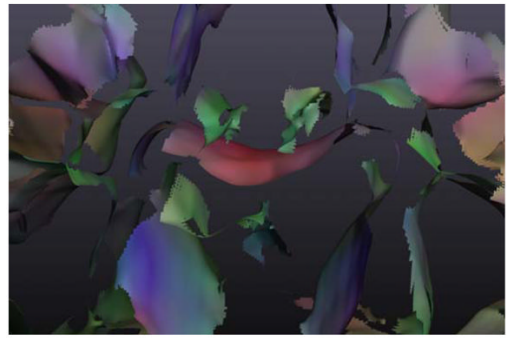

(b) Ridge Surfaces

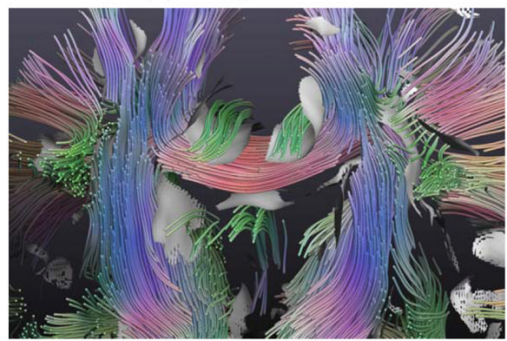

(d) Valleys with Fibers

Fig. 4.

Anisotropy Creases Near the Corpus Callosum. $\mathrm{CC}=$ corpus callosum; $\mathrm{IC}=$ internal capsule; $\mathrm{CR}=$ corona radiata; $\mathrm{FX}=$ fornix $\mathrm{CB}=$ cingulum bundles; $\mathrm{SFO}=$ superior fronto-occipital fasciculus; SLF = superior longitudinal fasciculus. 


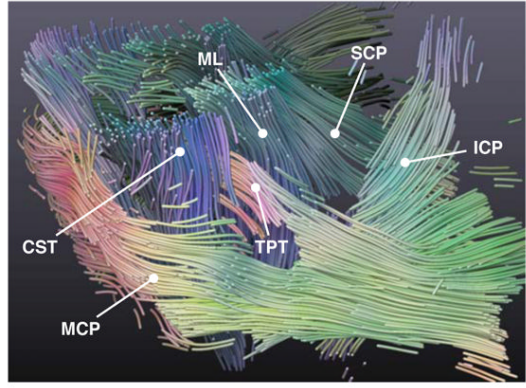

(a) RGB Map with Fibers

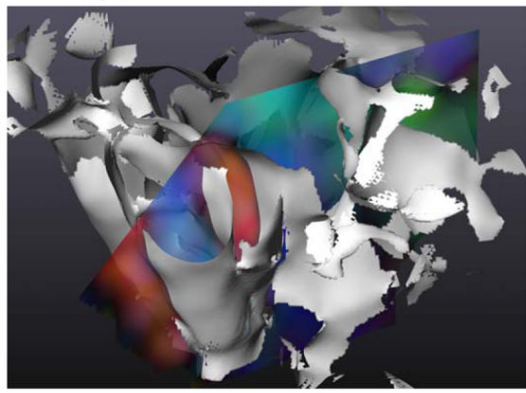

(c) Valley Surfaces

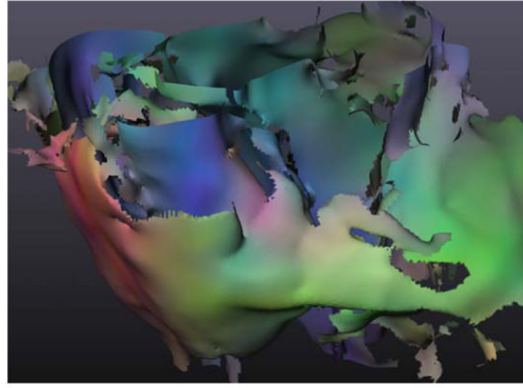

(b) Ridge Surfaces

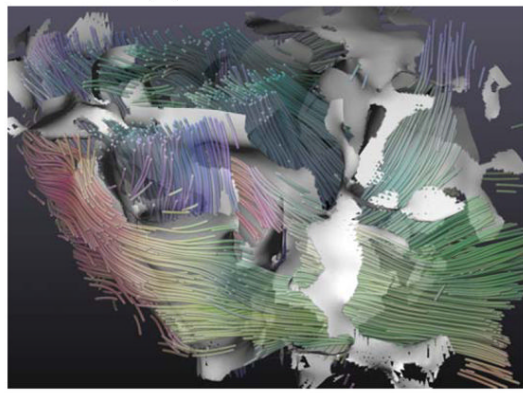

(d) Ridges and Valleys with Fibers

Fig. 5.

Anisotropy Creases in the Brainstem 


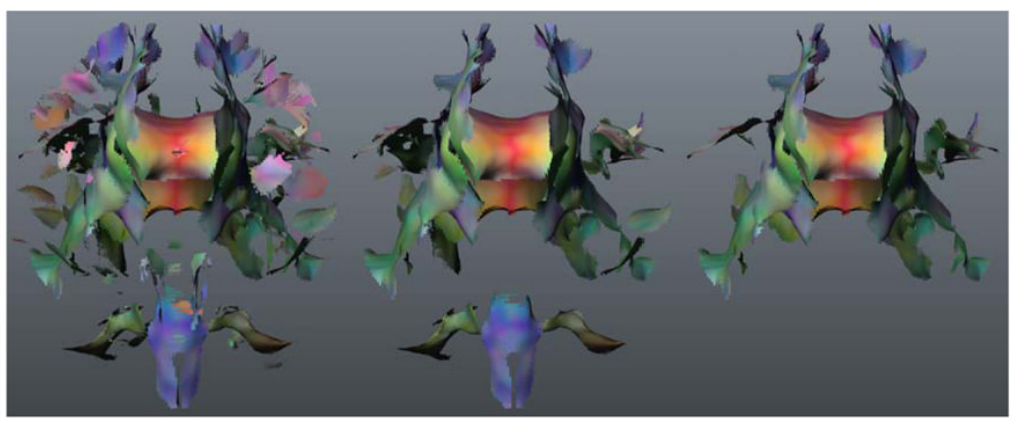

(a) Coronal view

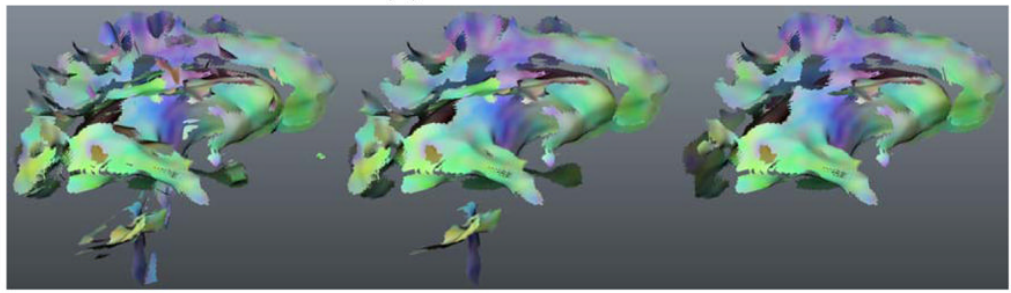

(b) Sagittal view

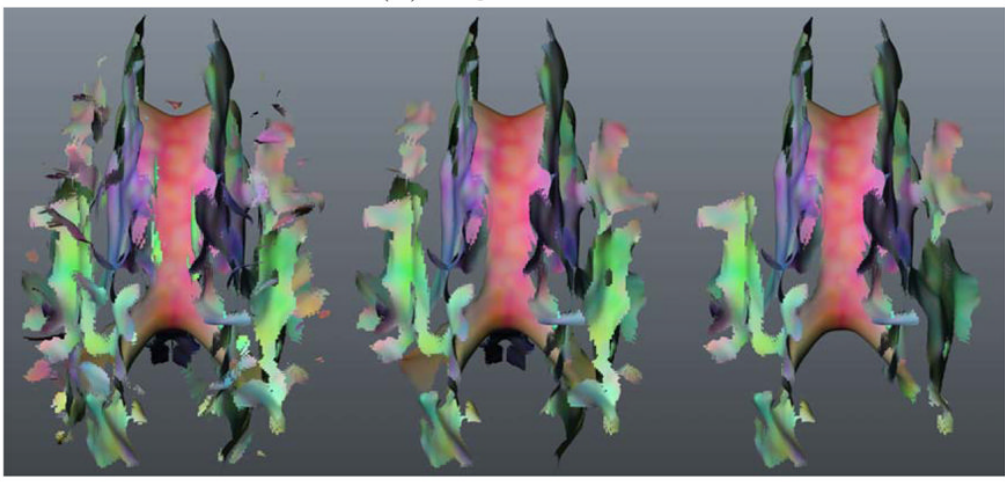

(c) Axial view

Fig. 6.

Ridge surface extraction results from a single scan, showing different numbers of connected components: all 742 (left), nine largest (middle), and single largest (right). 

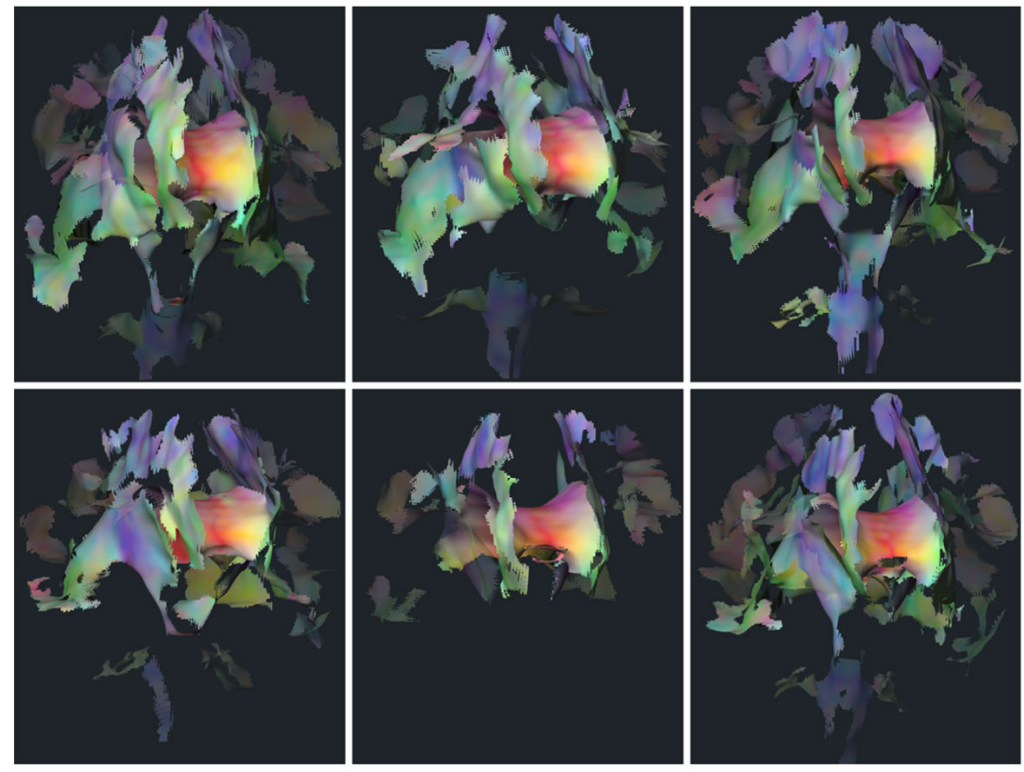

Fig. 7.

Ridge surface extraction results from six scans, viewed from an anterior (and slightly right) viewpoint, showing largest connected component (solid) and next eight largest CCs (translucent). 